\title{
Stochastic domination in space-time for the contact process
}

\section{Jacob van den Berg ${ }^{1,2}$ ～Stein Andreas Bethuelsen ${ }^{3}$}

${ }^{1}$ CWI, Amsterdam, The Netherlands

${ }^{2}$ VU University, Amsterdam,

The Netherlands

${ }^{3}$ Technical University of Munich,

München, Germany

\section{Correspondence}

Stein Andreas Bethuelsen, Department of Mathematics, Technical University of Munich, Boltzmannstr. 3, 85748

Garching, Germany.

Email: stein.bethuelsen@tum.de

\begin{abstract}
Liggett and Steif (2006) proved that, for the supercritical contact process on certain graphs, the upper invariant measure stochastically dominates an i.i.d. Bernoulli product measure. In particular, they proved this for $\mathbb{Z}^{d}$ and (for infection rate sufficiently large) $d$-ary homogeneous trees $T_{d}$. In this paper, we prove some space-time versions of their results. We do this by combining their methods with specific properties of the contact process and general correlation inequalities. One of our main results concerns the contact process on $T_{d}$ with $d \geq 2$. We show that, for large infection rate, there exists a subset $\Delta$ of the vertices of $T_{d}$, containing a "positive fraction" of all the vertices of $T_{d}$, such that the following holds: The contact process on $T_{d}$ observed on $\Delta$ stochastically dominates an independent spin-flip process. (This is known to be false for the contact process on graphs having subexponential growth.) We further prove that the supercritical contact process on $\mathbb{Z}^{d}$ observed on certain $d$-dimensional space-time slabs stochastically dominates an i.i.d. Bernoulli product measure, from which we conclude strong mixing properties important in the study of certain random walks in random environment.
\end{abstract}

\section{K EY W O R D S}

Bernoulli product measure, cone-mixing, contact process, downward FKG, stochastic domination 


\section{1 | INTRODUCTION AND MAIN RESULTS}

\section{1 | Background and outline of this paper}

Let $G=(V, E)$ be a connected graph of bounded degree, and let $\lambda \in(0, \infty)$. The contact process $\left(\eta_{t}\right)$ on $G$ with parameter $\lambda$ is the continuous-time interacting particle system on $\{0,1\}^{V}$ with local transition rates given by

$$
\eta \rightarrow \eta_{x} \text { at rate } \begin{cases}1, & \text { if } \eta(x)=1 \\ \lambda \sum_{\{y:\{x, y\} \in E\}} \eta(y), & \text { if } \eta(x)=0,\end{cases}
$$

for $x \in V$, and where $\eta_{x}$ is defined by $\eta_{x}(y):=\eta(y)$ for $y \neq x$, and $\eta_{x}(x):=1-\eta(x)$.

Equivalently (see also Section 2.2), one can imagine that for each site $x$ and each neighbor $y$ of $x$, there are "clocks," denoted by $I(y, x)$ and $H(x)$, which ring after independent, exponentially distributed, with mean $1 / \lambda$ and 1 respectively, times (independent of the other clocks). At each ring of the clock $I(y, x)$ the following happens: if $y$ has value 1 and $x$ has value 0 , the value of $x$ immediately changes to 1. At each ring of the clock $H(x)$ the following happens: if $x$ has value 1, the value of $x$ immediately changes to 0 .

The contact process was introduced by Harris [10] in 1974 as a toy model for the spread of an infection in a population. With this interpretation in mind, $\lambda$ is often referred to as the "infection" parameter and a site $x \in V$ is said to be infected at time $t$ if $\eta_{t}(x)=1$, otherwise it is said to be healthy. A central question is whether the infections "survive" with positive probability or eventually die out, that is, all sites become healthy. As general references on contact processes we mention the books [11] and [13] by Liggett, from which we next recall some well known properties.

The "healthy" configuration where all sites are equal to 0 , denoted by $\overline{0}$, is clearly an absorbing state for the contact process. On the other hand, starting from the full configuration where all sites are initially infected, the contact process evolves toward an invariant measure $\bar{\nu}_{\lambda}$. This state is often called the upper invariant measure. Throughout this text we write upper stationary contact process to denote the contact process whose law at an arbitrary time equals $\bar{\nu}_{\lambda}$.

A well-known property of the contact process is that, if $G$ is countable infinite, it undergoes a phase transition: there is a critical threshold $\lambda_{c} \in[0, \infty)$, depending on $G$, such that, for all $\lambda<\lambda_{c}, \bar{v}_{\lambda}=\delta_{\overline{0}}$, and for all $\lambda>\lambda_{c}, \bar{v}_{\lambda} \neq \delta_{\overline{0}}$. Here, $\delta_{\overline{0}}$ denotes the measure that concentrates on $\overline{0}$. In this paper, we focus on the supercritical phase, that is, the case $\lambda>\lambda_{c}$.

Van den Berg, Häggström, and Kahn [2] proved that the upper invariant measure satisfies the following property (called downward FKG in [15]): for any finite $\Delta \subset V$, the conditional measure $\bar{v}_{\lambda}(\cdot \mid \eta \equiv 0$ on $\Delta)$ is positively associated. (See Liggett [14] for a slightly stronger property). Liggett and Steif [15] used this result to show that, for the supercritical contact process on $\mathbb{Z}^{d}, d \geq 1$, the upper invariant measure stochastically dominates a non-trivial Bernoulli product measure (see Corollary 4.1 therein). For the $d$-ary homogeneous tree $T_{d}$, where each site has $d+1$ neighboring sites, they showed such a domination result for $\lambda>4$.

In this paper, we investigate if analogs of the mentioned domination results by Liggett and Steif hold for the contact process observed in space and time.

One of our main results concerns the upper stationary contact process on $T_{d}$ with $d \geq 2$. We show that, for $\lambda>\lambda_{c}(\mathbb{Z})$, there exists a subset $V$ of the vertices of $T_{d}$, containing a "positive fraction" of all the vertices of $T_{d}$, such that the following holds: the contact process on $T_{d}$ observed on $V$ stochastically dominates a non-trivial independent spin-flip process (see Section 1.2 for a definition of such processes). This is the content of Theorem 1.2 below. Interestingly, this cannot happen for the upper stationary contact process on graphs having subexponential growth (such as $\mathbb{Z}^{d}$ ), as shown in Proposition 1.1. 
We furthermore prove that the upper stationary contact process on $\mathbb{Z}^{d}$ with $d \geq 1$ and $\lambda>\lambda_{c}$, observed on certain (discrete-time) $d$-dimensional space-time slabs, stochastically dominates a nontrivial Bernoulli product measure. This is the content of Theorem 1.5 below. Using this, we conclude in Theorem 1.7 that the contact process projected onto a thin space-time slab satisfies a strong mixing property known as cone-mixing.

The projection of the contact process onto a sub-lattice can be interpreted as a hidden Markov model and is motivated for instance by the study of phase transition phenomena in nonlinear filtering (see Rebeschini and van Handel [17]) as well as the study of a random walk in a dynamic random environment (see Bethuelsen and Völlering [3]).

A key observation for our arguments is that the results in [2] imply that the above mentioned downward FKG property extends to the contact process observed in space-time (see Lemma 2.1). Our proofs are based on this observation, together with specific properties of the contact process and results and techniques from [15].

\section{Outline of this paper}

In the next Section, we recall some basic definitions before we present our main results for the contact process in Section 1.3. In Section 1.4, we discuss certain mixing properties which follow from our main results. Section 2 is devoted to some preliminary results. Proofs of our main results are provided in Section 3. In Section 4, we present some open questions.

\section{2 | Stochastic domination and Bernoulli product measures}

Besides the contact process there are two key concepts in the presentation of our main results, namely stochastic domination and Bernoulli product measures. For the convenience of the reader, we briefly recall their definitions.

Given a countable set $V$, we are interested in probability measures on $\Omega:=\{0,1\}^{V}$ and $D_{\Omega}[0, \infty)$, the set of càdlàg functions on $[0, \infty)$ taking values in $\Omega$. For this, denote by $\mathcal{F}$ the product $\sigma$-algebra corresponding to $\Omega$ and let $\mathcal{M}_{1}(\Omega)$ be the set of probability measures on $(\Omega, \mathcal{F})$, and similarly, let $\mathcal{M}_{1}\left(D_{\Omega}[0, \infty)\right)$ be the set of measures on $D_{\Omega}[0, \infty)$.

For $\rho \in[0,1]$, we denote by $\mu_{\rho} \in \mathcal{M}_{1}(\Omega)$ the Bernoulli product measure with density $\rho$. That is, for any finite $\Delta, \Lambda \subset V$ such that $\Delta \cap \Lambda=\emptyset$, the measure $\mu_{\rho}$ has cylinder probabilities given by

$$
\mu_{\rho}(\eta \in \Omega: \eta(x)=1 \forall x \in \Delta, \eta(x)=0 \forall x \in \Lambda)=\rho^{|\Delta|}(1-\rho)^{|\Lambda|} .
$$

A related object is the following continuous-time process. Given $\alpha \geq 0$, the independent spin-flip process $\left(\xi_{t}\right)$ with parameter $\alpha$ is the continuous-time Markov process on $\{0,1\}^{V}$ with local transition rates given by

$$
\eta \rightarrow \eta_{x} \text { at rate } \begin{cases}1, & \text { if } \eta(x)=1 ; \\ \alpha, & \text { if } \eta(x)=0,\end{cases}
$$

for $x \in V$. Note that $\left(\xi_{t}\right)$ is ergodic with unique invariant measure $\mu_{\rho}$, where $\rho=\rho(\alpha)=\alpha /(\alpha+1)$.

We next introduce the concept of stochastic domination. For this, we associate to $\Omega$ the partial ordering such that $\xi \leq \eta$ if and only if $\xi(x) \leq \eta(x)$ for all $x \in V$. An event $B \in \mathcal{F}$ is said to be increasing if $\xi \leq \eta$ implies $1_{B}(\xi) \leq 1_{B}(\eta)$. If $\xi \leq \eta$ implies $1_{B}(\xi) \geq 1_{B}(\eta)$ then $B$ is called decreasing. For $\mu_{1}, \mu_{2} \in \mathcal{M}_{1}(\Omega)$ we say that $\mu_{1}$ stochastically dominates $\mu_{2}$ if $\mu_{2}(B) \leq \mu_{1}(B)$ for all increasing events $B \in \mathcal{F}$. Recall that, by Strassen's theorem (see [11], p. 72), $\mu_{1}$ stochastically dominates $\mu_{2}$ is equivalent to the existence of a coupling $(\eta, \xi)$ so that $\eta$ has distribution $\mu_{2}$ and $\xi$ has 
distribution $\mu_{1}$, and $\eta \leq \xi$ a.s. The definition of stochastic domination readily translates to measures on $D_{\Omega}[0, \infty)$, by extending the partial ordering for elements in $\Omega$ to $\Omega^{[0, \infty)}$, requiring that $\xi_{t}(x) \leq \eta_{t}(x)$ for all $(x, t) \in V \times[0, \infty)$.

Another key concept used in the proof of the following theorems is that of downward FKG, to which we return to in Section 2.

\section{3 | Main results}

As shown in Liggett and Steif [15], Corollary 4.1, the upper stationary contact process on $\mathbb{Z}^{d}, d \geq 1$, with $\lambda>\lambda_{c}$ stochastically dominates a non-trivial Bernoulli product measure when observed at a fixed time $t$. That is, $\bar{v}_{\lambda}$ stochastically dominates $\mu_{\rho}$ for some $\rho \in(0,1)$.

On the other hand, as also shown in [15], stochastic domination of a non-trivial Bernoulli product measure does not hold in general for the entire space-time evolution. This can be extended to the contact process on graphs having subexponential growth.

Let $G=(V, E)$ be a connected graph of bounded degree and denote by $d: V \times V \rightarrow \mathbb{Z}_{\geq 0}$ the graph distance on G. Following [16, p. 181], the graph $G$ is said to have subexponential growth (of balls) if

$$
\liminf _{n \rightarrow \infty}|\{x \in V: d(o, x) \leq n\}|^{1 / n}=1, \quad \text { for some } o \in V
$$

where $|\cdot|$ denotes the cardinality. Otherwise $G$ is said to have exponential growth. Further, we say that $\Delta \subset V$ has positive density if

$$
\liminf _{n \rightarrow \infty} \frac{|\{x \in \Delta: d(o, x) \leq n\}|}{|\{y \in V: d(o, y) \leq n\}|}>0, \quad \text { for some } o \in V
$$

Remark 1.1 Since we assume that $G$ is connected and has bounded degree we may in (1.1) and (1.2) replace "for some $o \in V$ " by "for all $o \in V$."

Proposition 1.1 Let $\left(\eta_{t}\right)$ be the upper stationary contact process on a connected graph $G=(V, E)$ having subexponential growth and bounded degree with $\lambda>0$. Consider $\Delta \subset V$ having positive density. Then, for no parameter value except $\alpha=0$ can $\left(\eta_{t}\right)$ and $\left(\xi_{t}\right)$ be coupled so that, when initialized from $\bar{\nu}_{\lambda}$ and $\mu_{\rho(\alpha)}$ respectively, it holds that

$$
\widehat{\mathbb{P}}\left(\eta_{t}(x) \geq \xi_{t}(x) \text { for all }(x, t) \in \Delta \times[0, \infty)\right)=1
$$

The proof of Proposition 1.1 follows by an almost direct extension of the proof of [15, Proposition 1.1], and is given in Section 3.1. In fact, the proof also works if Condition (1.1) is replaced by the following condition,

$$
\liminf _{n \rightarrow \infty} \frac{|\{(x, y) \in E: d(o, x)=n=d(o, y)-1\}|}{|\{x \in V: d(o, x) \leq n\}|}=0, \text { for some } o \in V
$$

which is easily seen to be weaker than (1.1). A natural question is whether Proposition 1.1 also holds if (1.3) does not hold. Theorem 1.2 below states that this is not the case for homogeneous trees. See also Question 2 in Section 4.

Theorem 1.2 Let $\left(\eta_{t}\right)$ be the upper stationary contact process on $T_{d}, d \geq 2$, with $\lambda>\lambda_{c}(\mathbb{Z})$. Let $V$ be the set of vertices of $T_{d}$. Then there is a $\Delta \subset V$ having positive 
density together with an $\alpha=\alpha(\lambda)>0$ and a coupling $\widehat{\mathbb{P}}$ of $\left(\eta_{t}\right)$ and $\left(\xi_{t}\right)$, initialized from $\bar{\nu}_{\lambda}$ and $\mu_{\rho(\alpha)}$ respectively, such that

$$
\widehat{\mathbb{P}}\left(\eta_{t}(x) \geq \xi_{t}(x) \text { for all }(x, t) \in \Delta \times[0, \infty)\right)=1 .
$$

Thus, (1.4) in Theorem 1.2 concerns the contact process on $T_{d}$ projected onto a subset of $V \times[0, \infty)$ (a terminology we often refer to later). Theorem 1.2 says that the contact process projected onto $\Delta \times[0, \infty)$ stochastically dominates an independent spin-flip process.

To prove Theorem 1.2, we first show that the contact process on $\{0,1, \ldots\}$ observed at the vertex 0 stochastically dominates an independent spin-flip process (in fact, we show a generalization of this). Once this is obtained, Theorem 1.2 follows by a monotonicity argument. From the precise argument, given in Section 3.4, it moreover follows that the set $\Delta$ in Theorem 1.2 can be chosen such that the 1.h.s. of (1.2) equals $\frac{d-1}{d}$.

Denote by

$$
\tau^{x}:=\inf \left\{t \geq 0: \eta_{t}^{x} \equiv \overline{0}\right\}, \quad x \in V,
$$

the extinction time for the contact process $\left(\eta_{t}^{x}\right)$ started with only $x$ initially infected.

Theorem 1.3 Let $\left(\eta_{t}\right)$ be the upper stationary contact process on a connected graph $G=(V, E)$ having bounded degree with $\lambda>0$. Let $x \in V$ for which there exist $C, c>0$ such that,

$$
\begin{aligned}
& \mathbb{P}\left(\tau^{x}=\infty\right)>0 \\
& \mathbb{P}\left(s<\tau^{x}<\infty\right) \leq C e^{-c s}, \quad \text { for all } s \geq 0 .
\end{aligned}
$$

Then there exist $\alpha=\alpha(\lambda)>0$ and a coupling $\widehat{\mathbb{P}}$ of $\left(\eta_{t}\right)$ and $\left(\xi_{t}\right)$ initialized from $\bar{\nu}_{\lambda}$ and $\mu_{\rho(\alpha)}$ respectively, such that

$$
\widehat{\mathbb{P}}\left(\eta_{t}(x) \geq \xi_{t}(x) \text { for all } t \in[0, \infty)\right)=1 \text {. }
$$

Note that (1.6) and (1.7) are known to hold for all vertices throughout the supercritical phase for the contact process on $\mathbb{Z}^{d}, d \geq 1$ (see [13, Theorem 1.2.30]), and on $\{0,1, \ldots\}$ (see [7], p. 546 and [8]).

For the proof of Theorem 1.3 (in Section 3.2) we use that the contact process satisfies the downward FKG property in space-time (see Section 2 for a proper definition). Combining this with large deviation estimates of the probability that there are no infections at the site $x$ in the time interval $[0, t]$ and a general theorem in [15] (which we state in Lemma 2.3) yields the statement of Theorem 1.3.

It seems natural that Theorem 1.3 can be extended to the case where instead of observing the contact process at a single site, we observe it on a finite subset $\Delta \subset V$. Apart from some special cases, we are not able to show this in general. On the other hand, interestingly, we are able to extend Theorem 1.3 when restricting to observations at discrete times. For this, denote by

$$
\mathbb{Z}_{T}:=\{0, \pm T, \pm 2 T, \ldots\}, \quad T \in(0, \infty)
$$

Theorem 1.4 Let $\left(\eta_{t}\right), \lambda$ and $G$ be as in Theorem 1.3. Let $\Delta \subset V$ be finite and let $x \in \Delta$ be such that (1.6) and (1.7) hold. Then, for each $T \in(0, \infty)$, there exist 
$\rho=\rho(\lambda, T, \Delta)>0$ such that $\left(\eta_{t}\right)$ projected onto $\Delta \times \mathbb{Z}_{T}$ stochastically dominates a Bernoulli product measure with parameter $\rho$.

We end this section with a result, Theorem 1.5 below, for the supercritical contact process on $\mathbb{Z}^{d}$. As seen in Proposition 1.1, this process cannot stochastically dominate a non-trivial independent spin-flip process, not even when projected onto a subset $\Delta$ of positive density. This naturally leads to the question what happens for subsets $\Delta \subset \mathbb{Z}^{d}$ for which the 1.h.s. of (1.2) equals 0 . Theorem 1.5 concerns one such case, namely, the contact process projected onto certain (discrete-time) space-time slabs.

For $m \in \mathbb{N}$, let

$$
\mathbb{Z}_{d-1}^{d}(m):=\left\{\left(x_{1}, \ldots, x_{d}\right) \in \mathbb{Z}^{d}: x_{d} \in\{0, \ldots, m-1\}\right\}
$$

be the $(d-1)$-dimensional sublattice of $\mathbb{Z}^{d}$ of width $m$. When $m=1$ we simply write $\mathbb{Z}_{d-1}^{d}$.

Theorem 1.5 Let $\left(\eta_{t}\right)$ be the upper stationary contact process on $\mathbb{Z}^{d}, d \geq 1$, with $\lambda>\lambda_{c}$. Let $T \in(0, \infty)$ and $m \in \mathbb{N}$. Then there exists $\rho=\rho(\lambda, T, m)>0$ such that $\left(\eta_{t}\right)$ projected onto $\mathbb{Z}_{d-1}^{d}(m) \times \mathbb{Z}_{T}$ stochastically dominates a Bernoulli product measure with parameter $\rho$.

\section{4 | Mixing properties}

The purpose of this section is to show that the domination results we have obtained so far are useful in order to conclude mixing properties for the contact process, in particular when observed in a subspace.

We first note that, from the statement of Theorem 1.5 with $m=1$, we obtain a stronger notion of domination, which we present next. For $t \in(0, \infty)$ and $T \in(0, \infty)$, let $\mathbb{Z}_{T}(t):=\left\{s \in \mathbb{Z}_{T}: s<t T\right\}$

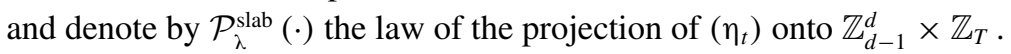

Corollary 1.6 Let $\left(\eta_{t}\right)$ be the upper stationary contact process on $\mathbb{Z}^{d}, d \geq 1$, with $\lambda>\lambda_{c}$. Let $T \in(0, \infty)$. Then, with $\rho=\rho(\lambda, T, 1)$ as in Theorem 1.5, for every finite $\Delta \subset$ $\mathbb{Z}_{d-1}^{d} \times \mathbb{Z}_{T}(0)$, the measure $\mathcal{P}_{\lambda}^{\text {slab }}(\cdot \mid \eta \equiv 0$ on $\Delta)$ stochastically dominates a Bernoulli product measure with density $\rho$ on $\mathbb{Z}_{d-1}^{d} \times\left(\mathbb{Z}_{T} \backslash \mathbb{Z}_{T}(0)\right)$.

Corollary 1.6 implies that the contact process projected on $\mathbb{Z}_{d-1}^{d} \times \mathbb{Z}_{T}$ has strong mixing properties. We next make precise what we mean by strong mixing properties.

Fix $T \in(0, \infty)$ and let, for $\theta \in\left(0, \frac{1}{2} \pi\right)$ and $t \geq 0$,

$$
C_{t}^{\theta}:=\left\{(x, s) \in \mathbb{Z}_{d-1}^{d} \times \mathbb{Z}_{T}:\|x\| \leq(s-t) \tan \theta\right\}
$$

be the cone whose tip is at $(o, t)$ and whose wedge opens up with angle $\theta$, where $o \in \mathbb{Z}^{d}$ denotes the origin. A process $\left(\xi_{t}\right)_{t \in \mathbb{Z}_{T}}$ on $\{0,1\}^{\mathbb{Z}_{d-1}^{d}}$ is said to be cone-mixing if, for all $\theta \in\left(0, \frac{1}{2} \pi\right)$,

$$
\lim _{t \rightarrow \infty} \sup _{\substack{A \in \mathcal{F}_{<0}, B \in \mathcal{F}_{t}^{\theta} \\ \mathbb{P}(A)>0}}|\mathbb{P}(B \mid A)-\mathbb{P}(B)|=0,
$$

where $\mathcal{F}_{<0}$ is the $\sigma$-algebra generated by the lower half-space $\left\{\xi_{s}(x):(x, s) \in \mathbb{Z}_{d-1}^{d} \times \mathbb{Z}_{T}(0)\right\}$ and $\mathcal{F}_{t}^{\theta}$ is the $\sigma$-algebra generated by $\left\{\xi_{s}(x):(x, s) \in C_{t}^{\theta}\right\}$. 
Theorem 1.7 Let $T \in(0, \infty)$. The upper stationary contact process on $\mathbb{Z}^{d}, d \geq 1$, with $\lambda>\lambda_{c}$, projected onto $\mathbb{Z}_{d-1}^{d} \times \mathbb{Z}_{T}$, is cone-mixing.

Cone-mixing was introduced in Comets and Zeitouni [6] and used there to prove limiting properties for certain random walks in mixing random environment. More recently, the cone-mixing condition has been adapted to random walks in dynamically evolving random environments, see Avena, den Hollander, and Redig [1]. For such models, a standing challenge is to prove limit properties for the random walk when the dynamic environment does not converge toward a unique stationary distribution, uniformly with respect to the initial state.

Theorem 1.7 gives one way to overcome this challenge for the particular case where the random environment is the contact process and the random walk stays inside $\mathbb{Z}_{d-1}^{d}$. Our result has recently been applied in Bethuelsen and Völlering [3] (see Theorem 2.6 therein) to prove (among other things) a law of large numbers for such random walks.

\section{2 | PRELIMINARIES}

In this section, we provide some preliminary results which are important for the proofs of our theorems.

\section{1 | Downward FKG and related properties}

As already mentioned, the concept of downward FKG (from now on abbreviated by dFKG) plays a key role in the proof of our main theorems. We next provide a definition of this and some related properties.

Definition 2.1 Let $\mu \in \mathcal{M}_{1}(\Omega)$. We say that $\mu$ is

a) positively associated if $\mu\left(B_{1} \cap B_{2}\right) \geq \mu\left(B_{1}\right) \mu\left(B_{2}\right)$ for any two increasing events $B_{1}, B_{2} \in \mathcal{F}$.

b) $d F K G$ iffor every finite $\Lambda \subset V$, the measure $\mu(\cdot \mid \eta \equiv 0$ on $\Lambda)$ is positively associated.

c) FKG iffor every finite $\Lambda \subset V$ and $\sigma \in \Omega$, the measure $\mu(\cdot \mid \eta \equiv \sigma$ on $\Lambda)$ is positively associated.

It is immediate that FKG implies $\mathrm{dFKG}$, which again implies positive association. The Bernoulli product measures $\mu_{\rho}, \rho \in[0,1]$, are examples of measures which clearly satisfy the FKG property. In [12] it was shown that the upper invariant measure is not always FKG, whereas [2] proved that it satisfies the dFKG property (see Theorem 3.3 and Equation (20) in that paper). With the same arguments as in [2] the latter property can be extended to the following lemma.

Lemma 2.1 Consider the upper stationary contact process $\left(\eta_{t}\right)$ on $G=(V, E)$ with $\lambda>0$. For any $t_{1}<t_{2}<\cdots<t_{n}$ the joint distribution of $\left(\eta_{t_{1}}, \ldots, \eta_{t_{n}}\right)$, which is a probability measure on $\Omega^{n}$, satisfies the $d F K G$ property.

Proof The proof is exactly the same as the proof of Theorem 3.3 in [2].

The following lemma gives a useful property, used in the proof of Theorem 1.4.

Lemma 2.2 Let $V$ be countable and assume that the random variables $\left(X_{i}\right)_{i \in V}$ are dFKG. Let $P=\left(P_{j}\right)_{j \geq 1}$ be a partitioning of $V$ into disjoint subsets. Then the random variables $\left(Y_{j}\right)_{j \geq 1}$ where $Y_{j}=\max \left\{X_{i}, i \in P_{j}\right\}$ are $d F K G$. 
Proof This follows easily from the dFKG property of $\left(X_{i}\right)$. (Use that the $Y_{j}$ 's are increasing functions of $\left(X_{i}\right)$ and that $\left.\left\{Y_{j}=0\right\}=\left\{X_{i}=0, i \in P_{j}\right\}\right)$.

The dFKG property was used in [15] to give a sufficient and necessary condition for a translation invariant measure $\mu$ on $\{0,1\}^{\mathbb{Z}}$ to dominate a Bernoulli product measure with density $\rho \in[0,1]$. Since their result plays an important role for our proofs, we recall the precise statement.

Lemma 2.3 (Theorem 1.2 in [15]) Let $V=\mathbb{Z}$ and let $\mu \in \mathcal{M}_{1}(\Omega)$ be a translation invariant measure on $\{0,1\}^{\mathbb{Z}}$ which is $d F K G$. Then the following are equivalent.

1. $\mu$ stochastically dominates $\mu_{\rho}$.

2. $\mu(\eta \equiv 0$ on $\{1,2, \ldots, n\}) \leq(1-\rho)^{n}$ for all $n$.

3. For all disjoint, finite subsets $\Lambda$ and $\Delta$ of $\{1,2,3, \ldots\}$, we have

$$
\mu(\eta(0)=1 \mid \eta \equiv 0 \text { on } \Lambda, \eta \equiv 1 \text { on } \Delta) \geq \rho .
$$

In [15] also a generalization of Lemma 2.3 to measures on $\{0,1\}^{Z^{d}}$ with $d \geq 2$ is presented. Though most of our arguments only use Lemma 2.3, for the proof of Corollary 1.6 we need the higher dimensional version, which we state below. We use the notation

$$
\mathcal{D}:=\left\{\left(x_{1}, \ldots, x_{d}\right) \in \mathbb{Z}^{d}: \exists m \text { such that } x_{i}=0 \forall i<m \text { and } x_{m}<0\right\}
$$

Lemma 2.4 (Theorem 4.1 in [15]) Let $V=\mathbb{Z}^{d}$ with $d \geq 2$ and let $\mu \in \mathcal{M}_{1}(\Omega)$ be a translation invariant measure on $\{0,1\}^{\mathbb{Z}^{d}}$ which is $d F K G$. Then the following are equivalent.

1. $\mu$ stochastically dominates $\mu_{\rho}$.

2. $\mu\left(\eta \equiv 0\right.$ on $\left.[1, n]^{d}\right) \leq(1-\rho)^{n^{d}}$ for all $n$.

3. For all disjoint, finite subsets $\Lambda$ and $\Delta$ of $\mathcal{D}$, we have

$$
\mu(\eta(o)=1 \mid \eta \equiv 0 \text { on } \Lambda, \eta \equiv 1 \text { on } \Delta) \geq \rho .
$$

Remark 2.1 Lemma 2.4 was stated (and proven) in [15] for $d=2$. However, the extension of their argument to general dimensions is immediate and yields Lemma 2.4 (as also commented directly before the proof in [15], see p. 232 therein).

\section{2 | The contact process}

We next give a brief and somewhat informal construction of the contact process via the so-called graphical representation. For a more thorough description we refer to [13], p. 32-34.

Let $G=(V, E)$ be a connected graph having bounded degree and fix $\lambda \in(0, \infty)$. Let $H:=$ $(H(x))_{x \in V}$ and $I:=(I(x, y))_{\{x, y\} \in E}$ be two independent collections of (doubly-infinite) i.i.d Poisson processes with rate 1 and $\lambda$, respectively. On $V \times \mathbb{R}$, draw the events of $H(x)$ as crosses over $x$ and the events of $I(x, y)$ as arrows from $x$ to $y$.

For $x, y \in V$ and $s \leq t$, we say that $(y, t)$ is connected to $(x, s)$ by a backwards path, written $(x, s) \leftarrow(y, t)$, if and only if there exists a directed path in $V \times \mathbb{R}$ starting at $(y, t)$, ending at $(x, s)$ and going either backwards in time without hitting crosses or "sideways" following arrows in the opposite 
direction of the prescribed direction. Otherwise we write $(x, s) \nleftarrow(y, t)$. In general, for $\Lambda, \Delta \subset V \times \mathbb{R}$, we write $\Delta \leftarrow \Lambda(\Delta \nleftarrow \Lambda)$ if there is a (there is no) backwards-path from $\Lambda$ to $\Delta$. Next, define the process $\left(\tilde{\eta}_{t}\right)$ on $\Omega$ by

$$
\tilde{\eta}_{t}(x):=\left\{\begin{array}{cc}
1, & \text { if } V \times\{-\infty\} \leftarrow(x, t) \\
0, & \text { otherwise }
\end{array}\right.
$$

where $V \times\{-\infty\} \leftarrow(x, t)$ denotes the event that there exists a backwards-path from $(x, t)$ to $V \times\{s\}$ for all $s \leq t$. It is well known that $\left(\tilde{\eta}_{t}\right)$ has the same distribution as the upper stationary contact process $\left(\eta_{t}\right)$ with infection parameter $\lambda>0$. In the following, we use the notation $\left(\eta_{t}\right)$ for either representations of the contact process and denote by $\mathcal{P}_{\lambda}$ the corresponding path measure.

We next state a lemma which is useful for most of our proofs. The proof and the statement is inspired by [5, Lemma 2.11]. For its statement, recall (1.5) and note that, as follows from the graphical representation,

$$
\mathcal{P}_{\lambda}\left(s<\tau^{x}<\infty\right)=\mathcal{P}_{\lambda}(V \times\{-s\} \leftarrow(x, 0) \text { but } V \times\{-\infty\} \nleftarrow(x, 0)) .
$$

Lemma 2.5 Consider the upper stationary contact process on a connected graph $G=(V, E)$ of bounded degree with $\lambda>0$. Let $\Delta \subset V$ and assume that there exist $\epsilon, C, c>0$ such that for all $x \in \Delta$,

$$
\begin{aligned}
& \mathcal{P}_{\lambda}\left(\tau^{x}=\infty\right)>\epsilon ; \\
& \mathcal{P}_{\lambda}\left(s<\tau^{x}<\infty\right) \leq C e^{-c s}, \quad s \geq 0
\end{aligned}
$$

Then, for any $T \in(0, \infty)$, there exists $\rho=\rho(T)>0$ such that for all $n$ and all $x_{1}, \ldots, x_{n} \in \Delta$

$$
\mathcal{P}_{\lambda}\left(\eta_{T i}\left(x_{i}\right)=0, i=1,2, \ldots, n\right) \leq(1-\rho)^{n}
$$

Proof Fix $T \in(0, \infty)$ and let $\mathbf{x}=\left(x_{i}\right)_{i \in \mathbb{Z}}$ be an infinite sequence of elements $x_{i} \in \Delta$. For $i \in \mathbb{Z}$, denote by

$$
D_{i}:=\inf \left\{l \in \mathbb{N}: V \times T(i-l) \nleftarrow\left(x_{i}, T i\right)\right\},
$$

and note that $D_{i} T$ yields an approximation (up to an error of at most $T$ ) on how far backwards in time $\left(x_{i}, T i\right)$ is connected to another space-time point. In particular, $\eta_{T i}\left(x_{i}\right)=$ 0 if and only if $D_{i}<\infty$.

Define $\mathcal{T}_{0}=0$ and, iteratively,

$$
\mathcal{T}_{i+1}:=\mathcal{T}_{i}+D_{n-\mathcal{T}_{i}}, \quad i \geq 0
$$

Let $K:=\sup \left\{i: \mathcal{T}_{i}<\infty\right\}$. We have the following relation (easy to check) between events:

$$
\begin{aligned}
\left\{\eta_{i T}\left(x_{i}\right)=0 \text { for } i=1, \ldots, n\right\} & =\left\{D_{1}, \ldots, D_{n}<\infty\right\} \\
& \subset\left\{\mathcal{T}_{K} \geq n\right\} .
\end{aligned}
$$


Finally, $\mathcal{P}_{\lambda}\left(\mathcal{T}_{K} \geq n\right)$ is exponentially small in $n$. This follows by standard arguments from the following consequences of (2.1) and (2.2) (using the independence properties of the graphical representation): for all $i$, all positive integers $t_{i}>t_{i-1}>\cdots>t_{1} \geq 1$, and all $s \geq 1$, we have

$$
\begin{aligned}
& \mathcal{P}_{\lambda}\left(D_{n-\mathcal{T}_{i}}=\infty \mid \mathcal{T}_{1}=t_{1}, \ldots, \mathcal{T}_{i}=t_{i}\right)>\epsilon \\
& \mathcal{P}_{\lambda}\left(s \leq D_{n-\mathcal{T}_{i}}<\infty \mid \mathcal{T}_{1}=t_{1}, \ldots, \mathcal{T}_{i}=t_{i}\right) \leq C e^{-c(s-1)}
\end{aligned}
$$

\section{3 | PROOFS}

\section{1 | Proof of Proposition 1.1}

For the proof of Proposition 1.1 we follow that of [15, Proposition 1.1], which we extend to graphs having subexponential growth.

Proof of Proposition 1.1 Let $G=(V, E)$ be a graph as in the statement of the proposition and let $\lambda \in(0, \infty)$. Fix $o \in V$, and consider $\Delta \subset V$ having positive density. Hence, there is a $\gamma>0$ and a $N \in \mathbb{N}$ such that, for all $n \geq N$, we have that $|\Delta \cap B(n)|>\gamma|B(n)|$, where $B(n):=\{x \in V: d(o, x) \leq n\}$.

Next, assume that the contact process on $G$ with infection parameter $\lambda>0$, projected onto $\Delta$, stochastically dominates a non-trivial independent spin-flip process with parameter $\alpha>0$. Consequently, for every $T>0$ and $n \geq N$, we have that

$$
\begin{aligned}
\mathcal{P}_{\lambda} & \left(\eta_{t}(x)=0 \text { for all }(x, t) \in B(n) \times[0, T]\right) \\
& \leq \mathcal{P}_{\lambda}\left(\eta_{t}(x)=0 \text { for all }(x, t) \in(\Delta \cap B(n)) \times[0, T]\right) \\
& \leq e^{-\gamma \alpha|B(n)| T}=e^{-c_{1}|B(n)| T}, \quad \text { where } c_{1}=\gamma \alpha .
\end{aligned}
$$

Thus, the probability in (3.1) decays exponentially at a rate proportional to the volume of $B(n) \times[0, T]$.

To conclude the statement of Proposition 1.1 for $\lambda>0$, we show that this estimate cannot hold and thus argue by means of contradiction. In doing so, we make use of the graphical representation of the contact process.

Let $A_{n, T}$ denote the event that there are no arrows in the graphical representation from sites outside $B(n)$ to any site in $B(n)$ during the time period $[0, T]$. Note that the 1.h.s. of (3.1) is bounded below by

$$
\mathcal{P}_{\lambda}\left(\left\{\eta_{0}(x)=0 \text { for } x \in B(n)\right\} \cap A_{n, T}\right)
$$

Moreover, this is again bounded below by

$$
\begin{gathered}
\mathcal{P}_{\lambda}\left(\left\{\eta_{0}(x)=0 \text { for } x \in B(n)\right\}\right) e^{-\lambda d|B(n+1) \backslash B(n)| T} \\
\geq\left[\prod_{x \in B(n)} \bar{v}_{\lambda}\left(\eta_{0}(x)=0\right)\right] e^{-\lambda d|B(n+1) \backslash B(n)| T},
\end{gathered}
$$

where $d$ denotes the maximum degree of $G$, and where we used that the contact process is positively associated. 
Next, since $G$ has subexponential growth (and hence satisfies (1.3)), we can find $n$ large such that $\lambda d|B(n+1) \backslash B(n)|<c_{1}|B(n)|$. For such $n$, by taking $T$ sufficiently large, the expression (3.2) is larger than the r.h.s. of (3.1): a contradiction.

\section{2 | Proof of Theorem 1.3}

Proof of Theorem 1.3 Consider the upper stationary contact process $\left(\eta_{t}\right)$ on a connected graph $G=(V, E)$ having bounded degree and with $\lambda>0$. Fix $x \in V$ such that (1.6) and (1.7) hold and define, for $t, s \in \mathbb{R}$ with $t<s$, the event $A_{t, s}:=\left\{\eta_{u}(x)=0: u \in[t, s)\right\}$. Further, let $f:[0, \infty] \times[0, \infty) \rightarrow[0,1]$ denote the function

$$
f(t, u)=\mathcal{P}_{\lambda}\left(A_{0, t} \mid A_{-u, 0}\right) .
$$

Clearly, $f(t, u)$ is non-increasing in $t$.

By Lemma 2.1 we have that, for each $n$, the collection of random variables $\left(\eta_{t}(y), y \in V, t \in \mathbb{Z}_{1 / n}\right)$ is dFKG (recall from (1.8) that $\mathbb{Z}_{1 / n}$ denotes $\{k / n: k \in \mathbb{Z}\}$ ). Further, it is standard (and easy to see) that, for $t<s$,

$$
\mathcal{P}_{\lambda}\left(A_{t, s}\right)=\lim _{n \rightarrow \infty} \mathcal{P}_{\lambda}\left(\eta_{u}(x)=0 \text { for all } u \in[t, s) \cap \mathbb{Z}_{1 / n}\right)
$$

Using this approximation, the above mentioned dFKG property, and general results for measures satisfying dFKG (see Section 2.1), it follows that

$$
f(t, u) \text { is non-decreasing in } u,
$$

so $f(t):=\lim _{u \rightarrow \infty} f(t, u)$ exists (and is $>0$ ) and $\mathcal{P}_{\lambda}\left(A_{0, t} \mid B\right) \leq f(t)$ for all events $B$ that are measurable with respect to $\left(\eta_{s}(x), s \leq 0\right)$. Further, since,

$$
\begin{aligned}
f(t+s, u) & =\mathcal{P}_{\lambda}\left(A_{0, t+s} \mid A_{-u, 0}\right) \\
& =\mathcal{P}_{\lambda}\left(A_{0, t} \mid A_{-u, 0}\right) \mathcal{P}_{\lambda}\left(A_{t, t+s} \mid A_{-u, t}\right) \\
& =f(t, u) f(s, t+u),
\end{aligned}
$$

we get, by letting $u \rightarrow \infty, f(t+s)=f(t) f(s)$, from which we obtain that there is a $c \geq 0$ such that

$$
f(t)=e^{-c t}, \quad \text { for all } t \geq 0 .
$$

By Lemma 2.5 (with $T=1$ ), there is an $\alpha>0$ such that

$$
\mathcal{P}_{\lambda}\left(A_{0, t}\right) \leq e^{-\alpha t}, \quad t \geq 1
$$

We claim that $c \geq \alpha$ (and hence $c>0$ ). The proof of this claim uses some of the arguments in the proof of Lemma 2.3 in [15]. For completeness, we include it here.

Suppose $c<\alpha$. Let $\alpha^{\prime} \in(c, \alpha)$. Fix $t>1$ and take an integer $l$ so large that $f(t, l t)$ is "very close" to $f(t)$ (and hence, by (3.4), to $e^{-c t}$ ). More precisely, we take $l$ sufficiently large so that

$$
f(t, l t)>e^{-\alpha^{\prime} t}
$$


For all integers $k \geq 0$ we have that, on the one hand (by (3.5)),

$$
\mathcal{P}_{\lambda}\left(A_{0, k l t}\right) \leq e^{-\alpha k l t}
$$

while on the other hand

$$
\begin{aligned}
\mathcal{P}_{\lambda}\left(A_{0, k l t}\right) & =\mathcal{P}_{\lambda}\left(A_{0, l t}\right) \prod_{i=l}^{k l-1} \mathcal{P}_{\lambda}\left(A_{i t,(i+1) t} \mid A_{0, i t}\right) \\
& \geq \mathcal{P}_{\lambda}\left(A_{0, l t}\right)(f(t, l t))^{k l} \\
& >\mathcal{P}_{\lambda}\left(A_{0, l t}\right) e^{-\alpha^{\prime} t k l}
\end{aligned}
$$

where the first inequality uses (3.3) and stationarity, and the second inequality comes from (3.6). Since $\alpha^{\prime}<\alpha$ (and $\mathcal{P}_{\lambda}\left(A_{0, l t}\right)>0$ ) this violates (3.7) if $k$ is sufficiently large, and yields a contradiction. This proves the claim.

By the claim, and the inequality one line below (3.3), we have that $\mathcal{P}_{\lambda}\left(A_{0, t} \mid B\right) \leq e^{-\alpha t}$ for all events $B$ that are measurable with respect to $\left(\eta_{s}(x), s \leq 0\right)$.

Finally, we also clearly have (by the contact process dynamics) that the conditional probability of the event $\left\{\eta_{s}(x)=1\right.$ for all $\left.s \in(0, t)\right\}$, given that $\eta_{0}(x)=1$ and any additional information about the process before time 0 , is exactly $e^{-t}$. We conclude that the process $\left(\eta_{s}(x)\right)$ dominates a spin-flip process which goes from state 0 to 1 at rate $\alpha$ and from 1 to 0 at rate 1 .

\section{3 | Proof of Theorem 1.4}

Proof of Theorem 1.4 Fix $T \in(0, \infty)$ and let $\Delta \subset V$ be finite with $x \in \Delta$ such that (1.6) and (1.7) hold. Furthermore, consider the doubly infinite sequence $\left(Y_{i}\right)_{i \in \mathbb{Z}}$, where $Y_{i}$ is given by

$$
Y_{i}:=\max \left\{\eta_{T i}(y): y \in \Delta\right\}
$$

By Lemmas 2.1 and 2.2 we note that $\left(Y_{i}\right)$ is $\mathrm{dFKG}$, and, since the upper stationary contact process is invariant under temporal shift, the sequence is also translation invariant. By Lemma 2.5 , there is a $\rho>0$ such that

$$
\mathcal{P}_{\lambda}\left(Y_{j}=0, j=1, \ldots n\right) \leq(1-\rho)^{n} .
$$

Hence, by Lemma 2.3, we get

$$
\mathcal{P}_{\lambda}\left(Y_{1}=1 \mid Y_{-j}=0, j=0, \ldots, n\right) \geq \rho .
$$

It is not difficult to see that (3.9) yields the following: for some $0<\tilde{\rho} \leq \rho$,

$$
\mathcal{P}_{\lambda}\left(\eta_{T}(x)=1 \text { for all } x \in \Delta \mid Y_{-j}=0, j=0, \ldots, n\right) \geq \tilde{\rho}
$$

for all $n \in \mathbb{N}$. Indeed, since the contact process evolves in continuous-time and the graph is connected, infections can spread with positive probability from any point in $\Delta$ to all other points in $\Delta$ in a small time interval. 
To make this more formal one can first consider a sequence defined similar to $\left(Y_{i}\right)$, only replacing $T$ by $T / 2$ in (3.8). By the same argument as above, using again the $\mathrm{dFKG}$ property, we have that for some $\delta>0$,

$$
\mathcal{P}_{\lambda}\left(\max \left\{\eta_{T / 2}(y): y \in \Delta\right\}=1 \mid Y_{-j}=0, j=0, \ldots, n\right)>\delta
$$

Furthermore, since $\Delta$ is finite, and $G$ is connected, there is an $\epsilon>0$ such that (with the notation introduced below (1.5))

$$
\inf _{z \in \Delta} \mathcal{P}_{\lambda}\left(\eta_{\frac{T}{2}}^{z}(y)=1 \text { for all } y \in \Delta\right)>\epsilon
$$

Thus, using the fact that the contact process is a Markov process, we conclude (3.10) with $\tilde{\rho} \geq \epsilon \delta>0$.

Finally, using again the dFKG property of the collection $\left(\eta_{T i}(y), y \in \Delta, i \in \mathbb{Z}\right)$, we obtain that (3.10) still holds if the conditioning $\left\{Y_{-j}=0, j=0, \ldots, n\right\}$ is replaced by any event measurable with respect to $\left(\eta_{-T i}(y), y \in \Delta, i \geq 0\right)$. This concludes the proof of the theorem.

\section{4 | Proof of Theorem 1.2}

To prove Theorem 1.2, we first prove that the contact process on $\{0,1, \ldots\}$ observed at the vertex $\{0\}$ stochastically dominates an independent spin-flip process. Indeed, the required estimates (1.6) and (1.7) for this context is provided by the following result in [7], see Equation (21) on page 546 therein.

Lemma 3.1 ([7], Equation(21), and [8]) Consider the contact process on $V=\{0,1, \ldots\}$ with $\lambda>\lambda_{c}$. Then there exists constants $\epsilon, C, c>0$ such that (1.6) and (1.7) hold.

Proof of Theorem 1.2 First, by Lemma 3.1 applied to Theorem 1.3, we have that the contact process on $\{0,1,2, \ldots\}$ with $\lambda>\lambda_{c}$ observed at the vertex $\{0\}$ stochastically dominates an independent spin flip process with $\alpha>0$.

From the above observation, the statement of Theorem 1.2 follows by a monotonicity argument using again the graphical construction of the contact process.

To make this last argument precise, fix an arbitrary point $o \in T_{d}$ and call it the root. Denote by $u(o)=0$ its label. Furthermore, label the remaining sites according to their distance with respect to $o$ in a unique way. That is, each $x \in T_{d}$ with $\|x-o\|=1$ has a label $u(x)=(0, i)$ for some $i \in\{1, \ldots, d+1\}$ and for $y \in T_{d}$ satisfying $\|y-o\|=$ $\|z-o\|+1=n$ and $\|y-z\|<n, n \geq 2$, set $u(y)=(u(z), i), i \in\{1, \ldots, d\}$. Thus, for each $x \in T_{d} \backslash\{o\}$, we have that

$$
u(x) \in \bigcup_{n \geq 0}\left[\{0\} \times\{1, \ldots, d+1\} \times\{1, \ldots, d\}^{n}\right] .
$$

Denote by $\Delta \subset T_{d}$ the set of vertices having as last entry of its label a number different from 1. Using the graphical representation of the contact process, consider the process $\left(\xi_{t}\right)$ on $T_{d}$ where for each $(x, t) \in \Delta \times \mathbb{R}$ we set $\xi_{t}(x)=1$ if and only if there is an infinite backwards path from $(x, t)$ constrained to infection arrows between the sites with label $\{u(x),(u(x), 1),(u(x), 1,1), \ldots\}$. Moreover, for $x \in \Delta^{c}$, let $\xi_{t}(x)=0$ for all $t \in \mathbb{R}$. 
By construction, the evolution of $\left(\xi_{t}\right)$ on $T_{d}$ is dominated by that of the contact process. Furthermore, the evolution at site $x \in \Delta$ is in one-to-one correspondence with the contact process on $\{0,1,2, \ldots\}$, and the evolution at different sites $x, y \in \Delta$ is independent. Thus, on the set $\Delta$ the process $\left(\xi_{t}\right)$ stochastically dominates a non-trivial independent spin-flip process, and consequently, so does also the contact process. Last, we note that, from the above construction, it holds that $\Delta$ has positive density and that the 1.h.s. of (1.2) equals $\gamma=\frac{d-1}{d}>0$. This concludes the proof.

\section{5 | Proof of Theorem 1.5}

In order to prove Theorem 1.5, we make use of the well known fact that the supercritical contact process on $\mathbb{Z}^{d}$ with $d \geq 2$ survives in 2-dimensional space-time slabs (see [4]). More precisely, let, for $k \in \mathbb{N}$,

$$
S_{k}:=\left\{x \in \mathbb{Z}^{d}: x_{i} \in\{0,1, \ldots, k-1\}, i=1, \ldots d-1\right\},
$$

and denote by $\left({ }_{k} \eta_{t}\right)$ the contact process on $S_{k}$ and by $\mathcal{P}_{\lambda, k}$ its path measure. This process on $S_{k}$ with $\lambda>\lambda_{c}\left(\mathbb{Z}^{d}\right)$ survives with positive probability if the width $k$ is large enough. The proof of this proceeds via a block argument and comparison with a certain 2-dimensional (dependent) directed percolation model. This argument also gives a form of exponential decay, more precisely, the following lemma holds.

Lemma 3.2 Let $\lambda>\lambda_{c}\left(\mathbb{Z}^{d}\right)$ and $d \geq 2$. Then there exists $k \in \mathbb{N}$ and $\epsilon, C, c \in(0, \infty)$, such that for all $x \in S_{k}$,

$$
\begin{aligned}
& \mathcal{P}_{\lambda, k}\left(\tau^{x}=\infty\right)>\epsilon ; \\
& \mathcal{P}_{\lambda, k}\left(s<\tau^{x}<\infty\right) \leq C e^{-c s}, \quad \text { for all } s>0 .
\end{aligned}
$$

Proof This follows again by comparison with a 2-dimensional directed percolation model and a renormalization arguments. For a proof we refer the reader to the proof of Theorem 1.2.30a) in [13], where such an argument is explained in detail. Though proved there for the unrestricted contact process $\left(\eta_{t}\right)$ the argument works, mutatis mutandis, for $\left({ }_{k} \eta_{t}\right)$ as soon as $k$ is taken sufficiently large.

Proof of Theorem 1.5 Fix $T \in(0, \infty)$ and note that the case $d=1$ is an immediate consequence of Theorem 1.4. Indeed, the estimates (1.6) and (1.7) for that case are known to hold due to [8, Theorem 5].

For the case $d \geq 2$ we use a slightly more involved argument, by partitioning $\mathbb{Z}^{d} \times \mathbb{R}$ into slabs. Fix $k$ such that (3.11) and (3.12) hold. For $\mathbf{i}=\left(i_{1}, \ldots, i_{d-1}\right) \in \mathbb{Z}^{d-1}$, let $P_{\mathbf{i}}=\left(S_{k}+k \cdot\left(i_{1}, \ldots, i_{d-1}, 0\right)\right) \times \mathbb{R}$. Note that $P_{\mathbf{i}} \cap P_{\mathbf{j}}=\emptyset$ whenever $\mathbf{i} \neq \mathbf{j}$ and that $\bigcup_{\mathbf{i} \in \mathbb{Z}^{d-1}} P_{\mathbf{i}}=\mathbb{Z}^{d} \times \mathbb{R}$.

Next, consider the process $\left(\zeta_{t}\right)$ which is obtained from the graphical representation of the contact process on $\mathbb{Z}^{d}$ by suppressing all infection arrows between slabs $P_{\mathbf{j}}$. Trivially the evolution of $\left(\zeta_{t}\right)$ is dominated by that of $\left(\eta_{t}\right)$. Moreover, the evolution of $\left(\zeta_{t}\right)$ in each slab is independent of the others and has the same law as $\left({ }_{k} \eta_{t}\right)$.

Let $\mathbf{i} \in \mathbb{Z}^{d-1}$. By applying Theorem 1.4 with $\Delta=\mathbb{Z}_{d-1}^{d}(m) \cap\left(S_{k}+k \cdot(\mathbf{i}, o)\right)$, it follows that the process $\left(\zeta_{t}\right)$ observed on the vertices $\Delta$ at times that are multiples of $T$ stochastically dominates a non-trivial Bernoulli product measure with density $\rho>0$. By 
the above mentioned independence, this implies the statement of Theorem 1.5 for $\left(\zeta_{t}\right)$. Since $\left(\zeta_{t}\right)$ is stochastically dominated by $\left(\eta_{t}\right)$, we conclude the proof.

\section{6 | Proof of Corollary 1.6}

Proof of Corollary 1.6 Fix $T \in(0, \infty)$ and recall the definition of $\mathcal{P}_{\lambda}^{\text {slab }}$ in Section 1.4. Note that $\mathcal{P}_{\lambda}^{\text {slab }}$ is translation invariant and that, due to Lemma 2.1, it is also dFKG. In particular, we may apply Lemma 2.4 to $\mathcal{P}_{\lambda}^{\text {slab }}$.

A direct consequence of Theorem 1.5 with $m=1$ is that whenever $\lambda>\lambda_{c}$, there is a $\rho>0$ such that

$$
\mathcal{P}_{\lambda}^{\text {slab }}\left(\eta_{s}(x)=0,(x, s) \in \mathbb{Z}_{d-1}^{d} \times \mathbb{Z}_{T} \cap[1, n]^{d} \times[T, n T]\right) \leq(1-\rho)^{n^{d}} .
$$

Hence, the measure $\mathcal{P}_{\lambda}^{\text {slab }}$ satisfies Property 2 in Lemma 2.4. Consequently, $\mathcal{P}_{\lambda}^{\text {slab }}$ also satisfies Property 3 in Lemma 2.4, from which the statement of Corollary 1.6 follows.

\section{7 | Proof of Theorem 1.7}

Theorem 1.7 follows from Corollary 1.6 and a standard coupling argument, together with classical properties of the contact process.

Proof of Theorem 1.7 Fix $T \in(0, \infty)$ and let $\rho>0$ be such that the statement of Corollary 1.6 holds. Next, denote by $\mu \in \mathcal{M}_{1}(\Omega)$ the probability measure under which all vertices outside $\mathbb{Z}_{d-1}^{d}$ have value 0 a.s., and those in $\mathbb{Z}_{d-1}^{d}$ correspond with independent Bernoulli random variables with parameter $\rho$. Further, for $\eta \in \Omega$, denote by $\delta_{\eta} \in \mathcal{M}_{1}(\Omega)$ the probability measure which concentrates on $\eta$, and write $\overline{1} \in \Omega$ for the configuration where all sites are equal to 1 . Then, by Corollary 1.6, and since $\bar{v}_{\lambda} \leq \delta_{\overline{1}}$, we have, for $\theta \in(0, \pi / 2), t>0$ and $B \in \mathcal{F}_{t}^{\theta}$ increasing, and for any $A \in \mathcal{F}_{<0}$ with $\mathcal{P}_{\lambda}^{\text {slab }}(A)>0$, that

$$
\left|\mathcal{P}_{\lambda}^{\text {slab }}(B \mid A)-\mathcal{P}_{\lambda}^{\text {slab }}(B)\right| \leq \widehat{\mathcal{P}}_{\mu, \delta_{\overline{1}}}\left(\eta^{1} \neq \eta^{2} \text { on } C_{t}^{\theta}\right),
$$

where $\widehat{\mathcal{P}}_{\mu, \delta_{\overline{1}}}$ is the standard graphical construction coupling of the contact processes on $\mathbb{Z}^{d}$ started at time 0 from a configuration drawn according to $\mu$ and $\delta_{\overline{1}}$, respectively.

Furthermore, we have that

$$
\begin{aligned}
\widehat{\mathcal{P}}_{\mu, \delta_{\overline{1}}}\left(\eta^{1} \neq \eta^{2} \text { on } C_{t}^{\theta}\right) & \leq \sum_{(x, s) \in C_{t}^{\theta}} \widehat{\mathcal{P}}_{\mu, \delta_{\overline{1}}}\left(\eta_{s}^{1}(x) \neq \eta_{s}^{2}(x)\right) \\
& =\sum_{(x, s) \in C_{t}^{\theta}} \widehat{\mathcal{P}}_{\mu, \delta_{\overline{1}}}\left(\eta_{s}^{1}(o) \neq \eta_{s}^{2}(o)\right),
\end{aligned}
$$

where the last equation holds due to translation invariance in the first $(d-1)$ spatial directions.

Since the set of increasing events in $\mathcal{F}_{t}^{\theta}$ generates $\mathcal{F}_{t}^{\theta}$, in order to conclude the argument, it is sufficient to show that, for some $C, c \in(0, \infty)$, we have

$$
\widehat{\mathcal{P}}_{\mu, \delta_{\overline{1}}}\left(\eta_{s}^{1}(o) \neq \eta_{s}^{2}(o)\right) \leq C e^{-c s} .
$$


This can be shown using known estimates for the supercritical contact process on $\mathbb{Z}^{d}$. For completeness we present the details.

Let $N:=\inf \left\{\|x\|: \eta_{0}^{1}(x)=1\right.$ and $\left.\tau^{x}=\infty\right\}$. Then, for any $a>0$, we have that

$$
\begin{aligned}
\widehat{\mathcal{P}}_{\mu, \delta_{\overline{1}}}\left(\eta_{s}^{1}(o) \neq \eta_{s}^{2}(o)\right) \leq & \widehat{\mathcal{P}}_{\mu_{\rho}, \delta_{\overline{1}}}(\{N>a s\}) \\
& +\widehat{\mathcal{P}}_{\mu_{\rho}, \delta_{\overline{1}}}\left(\left\{\eta_{s}^{1}(o) \neq \eta_{s}^{2}(o)\right\} \cap\{N \leq a s\}\right) .
\end{aligned}
$$

That the first term on the righthand side decays exponentially (in as) follows from [13, Theorem 1.2.30]. For the other term, we have that

$$
\begin{aligned}
& \widehat{\mathcal{P}}_{\mu_{\rho}, \delta_{\overline{1}}}\left(\left\{\eta_{s}^{1}(o) \neq \eta_{s}^{2}(o)\right\} \cap\{N \leq a s\}\right) \\
& \quad \leq \sum_{y \in[-a s, a s]^{d}} \widehat{\mathcal{P}}_{\delta_{\overline{0}_{y}}, \delta_{\overline{1}}}\left(\left\{\eta_{s}^{1}(o) \neq \eta_{s}^{2}(o)\right\} \cap\left\{\tau^{y}=\infty\right\}\right) \\
& \quad \leq \sum_{y \in[-a s, a s]^{d}} \widehat{\mathcal{P}}_{\delta_{\overline{0}_{y}}, \delta_{\overline{1}}}\left(\eta_{s}^{1}(o) \neq \eta_{s}^{2}(o) \mid \tau^{y}=\infty\right),
\end{aligned}
$$

where $\overline{0}_{y}$ is the configuration given by $\overline{0}_{y}(x)=\overline{0}(x)=0$ for all $y \neq x$ and $\overline{0}_{y}(y)=$ $1-\overline{0}(y)=1$. From the large deviation estimates obtained in [9, Theorem 1.4], by choosing $a>0$ in (3.17) sufficiently small, the term inside the sum of (3.17) decays exponentially (in $s$ ), uniformly for $y \in[-a s, a s]^{d}$. Hence, since the sum only contains polynomially many terms, we have that (3.16) decays exponentially with respect to $s$.

In conclusion, there exist $C, c>0$ such that (3.15) holds, from which, by (3.13) and (3.14), we conclude the proof.

\section{4 | OPEN QUESTIONS}

We expect that the statement of Theorem 1.2 can be improved.

Question 1 Can the condition $\lambda>\lambda_{c}(\mathbb{Z})$ in Theorem 1.2 be replaced by $\lambda>\lambda_{c}\left(T_{d}\right)$ ?

Question 2 Does Theorem 1.2 hold with $\Delta=T_{d}$ ?

Motivated by Theorem 1.5 and Lemma 2.5, the following questions seem natural.

Question 3 Consider the upper stationary contact process on $\mathbb{Z}^{d}, d \geq 1$, with $\lambda>\lambda_{c}$, and let $\mathbf{x}=\left(x_{i}\right)_{i \in \mathbb{Z}}$ be an infinite sequence of elements in $\mathbb{Z}^{d}$. Does the contact process projected onto $\left\{\left(x_{i}, i\right): i \in \mathbb{Z}\right\}$ stochastically dominate a non-trivial Bernoulli product measure?

Question 4 Consider the upper stationary contact process $\left(\eta_{t}\right)$ on $\mathbb{Z}^{d}, d \geq 1$, with $\lambda>\lambda_{c}$, and let $\mathbf{X}=\left(X_{i}\right)_{i \geq 0}$ be a simple random walk on $\mathbb{Z}^{d}$ started at $X_{0}=o$. Does the sequence $\left(\eta_{i}\left(X_{i}\right)\right)_{i \geq 0}$ dominate a non-trivial Bernoulli sequence?

Remark 4.1 A positive answer to Question 3 with a uniform bound on the density $\rho>0$ would imply a positive answer to Question 4. 
Lastly, motivated by Proposition 1.1 and Theorem 1.5, we state the following question.

Question 5 Consider the upper stationary contact process $\left(\eta_{t}\right)$ on $\mathbb{Z}^{d}, d \geq 1$, with $\lambda>\lambda_{c}$. For which $\Delta \subset \mathbb{Z}^{d}$ having "zero density" (ie, the 1.h.s. of (1.2) equals 0 ) does $\left(\eta_{t}\right)$ projected onto $\Delta \times[0, \infty)$ dominate a non-trivial independent spin-flip process?

\section{ACKNOWLEDGMENT}

The authors thank Markus Heydenreich and Matthias Birkner for discussions and comments. S.A. Bethuelsen thanks LMU Munich for hospitality during the writing of the paper. S.A. Bethuelsen was supported by the Netherlands Organization for Scientific Research (NWO).

\section{REFERENCES}

[1] L. Avena, F. den Hollander, and F. Redig, Law of large numbers for a class of random walks in dynamic random environments, Electron. J. Probab. 16 (2011), no. 21, 587-617.

[2] J. van den Berg, O. Häggström, and J. Kahn, Some conditional correlation inequalities for percolation and related processes, Random Struct. Algorithms 29 (2006), no. 4, 417-435.

[3] S. A. Bethuelsen and F. Völlering, Absolute continuity and weak uniform mixing of random walk in dynamic random environment, Electron. J. Probab. 21 (2016), no. 71, 32 p.

[4] C. Bezuidenhout and G. Grimmett, The critical contact process dies out, Ann. Probab. 18 (1990), no. 4, 1462-1482.

[5] M. Birkner, J. Černý, and A. Depperschmidt, Random walks in dynamic random environments and ancestry under local population regulation, Electron. J. Probab. 21 (2016), no. 38, 1-43.

[6] F. Comets and O. Zeitouni, A law of large numbers for random walks in random mixing environments, Ann. Probab. 32 (2004), no. 1B, 880-914.

[7] R. Durrett and D. Griffeath, Contact processes in several dimensions, Z. Wahrsch. Verw. Gebiete 4 (1982), no. 59, $535-552$.

[8] R. Durrett and D. Griffeath, Supercritical contact processes on $\mathbb{Z}$, Ann. Probab. 11 (1983), no. 1, 1-15.

[9] O. Garet and R. Marchand, Large deviations for the contact process in random environment, Ann. Probab. 42 (2014), no. 4, 1438-1479.

[10] T. E. Harris, Contact interactions on a lattice, Ann. Probab. 2 (1974), 969-988.

[11] T. M. Liggett, Interacting particle systems, Springer, New York, 1985.

[12] T. M. Liggett, Survival and coexistence in interacting particle systems, Probability and phase transition (G. Grimmett, ed.), Kluwer Academics, Dordrecht, 1994.

[13] T. M. Liggett, Stochastic interacting systems: Contact, voter and exclusion processes, Springer, Berlin, 1999.

[14] T. M. Liggett, Conditional association and spin systems, ALEA Lat. Am. J. Probab. Math. Stat. 1 (2006), 1-19.

[15] T. M. Liggett and J. E. Steif, Stochastic domination: the contact process, Ising models and FKG measures, Ann. Inst. H. Poincaré Probab. Statist. 42 (2006), no. 2, 223-243.

[16] R. Lyons and Y. Peres, Probability on trees and networks, Cambridge University Press, New York, 2016. available at http://pages.iu.edu/ ${ }^{\text {rdlyons / }}$

[17] P. Rebeschini and R. van Handel, Phase transitions in nonlinear filtering, Electron. J. Probab. 20 (2015), no. 7, $1-46$.

How to cite this article: van den Berg J, Bethuelsen SA. Stochastic domination in space-time for the contact process. Random Struct Alg. 2018;00:1-17. https://doi.org/10.1002/rsa.20766 